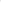

\title{
The effects of combined balance and complex training versus complex training only on measures of physical fitness in young female handball players
}

Running head: Balance and complex training in female players

Helmi Chaabene ${ }^{1,2^{*}}$, Yassine Negra $^{3 *}$, Jason Moran ${ }^{4}$, Senda Sammoud ${ }^{3}$, Rodrigo RamirezCampillo ${ }^{5}$, Urs Granacher ${ }^{1}$, Olaf Prieske ${ }^{6}$

${ }^{1}$ Division of Training and Movement Sciences, Research Focus Cognition Sciences, University of Potsdam, Am Neuen Palais 10, 14469 Potsdam, Germany. ${ }^{2}$ High Institute of Sports and Physical Education, Kef, University of Jendouba, Tunisia. ${ }^{3}$ Research Unit "Sports Performance, Health \& Society" Higher Institute of Sports and Physical Education of Ksar Said, University of Manouba, Tunis, Tunisia. ${ }^{4}$ School of Sport, Rehabilitation and Exercise Sciences, University of Essex, Colchester, Essex, United Kingdom. ${ }^{5}$ Human Performance Laboratory. Department of Physical Activity Sciences. Universidad de Los Lagos. Osorno, Chile. ${ }^{6}$ Division of Exercise and Movement, University of Applied Sciences for Sports and Management Potsdam, Potsdam, Germany

\section{Corresponding author}

Helmi Chaabene Ph.D

Division of Training and Movement Sciences, Research Focus Cognitive Sciences, University of Potsdam, Am Neuen Palais 10, 14469 Potsdam, Germany. High Institute of Sports and Physical Education, Kef, University of Jendouba, Tunisia

Email: chaabanehelmi@hotmail.fr

*denotes equal contribution

Abstract: 235

Text only: 3598

Tables: 3

Figure: 2

References: 31 (2) 


\section{Abstract}

Purpose: To examine the effects of balance exercises conducted prior to complex training (bCT) vs. complex training (CT) only on measures of physical fitness in young female elite handball players. Methods: Participants aged 17 years were randomly assigned to bCT $(n=11)$ or CT ( $n=12)$. The two training interventions lasted eight weeks with two sessions per week in replacement of some technical/tactical handball exercises and were matched for total training volume. Before and after training, tests were performed for the evaluation of proxies of muscle power (countermovement-jump [CMJ] height, standing-long-jump [SL] distance, and reactive-strength-index $[\mathrm{RSI}]$ ), muscle strength (back half-squat one-repetition maximum [1RM]), dynamic balance (Y-balance test), linear sprint speed (20-m sprint test), and changeof-direction speed (T-test). Results: Two-factor repeated measures ANOVA revealed significant group $\times$ time interactions for the RSI $(d=0.99, p=0.03)$ and Y-balance test score $(d=1.32, p<0.01)$. Post-hoc analysis indicated significant pre-to-post RSI improvements in CT $(d=0.69, p=0.04)$ only. For the $Y$-balance test, significant pre-to-post increases were found in bCT ( $d=0.71, p=0.04)$ with no significant changes in CT (d=0.61, $p=0.07)$. Additionally, significant main effects of time were observed for half-squat 1RM, CMJ, SL, and T-test performances ( $d=1.50$ to $3.10, p<0.05$ ). Conclusion: Both $\mathrm{BCT}$ and CT interventions were effective in improving specific measures of physical fitness in young elite female handball players. If the training goal is to additionally improve balance, balance exercises can be conducted within a CT training session and prior to CT exercises.

Key words: Strength training, plyometric exercise, girls, team sports. 


\section{Introduction}

Both, strength and plyometric training as single-mode resistance training types have proven to be effective to enhance components of physical fitness (e.g., muscle strength, muscle power, linear sprint speed), irrespective of age and sex. ${ }^{1,2}$ If sequenced in one exercise session, there is evidence that the performance of strength exercises before jump exercises has the potential to acutely enhance subsequent jump performance, a phenomenon known as postactivation performance enhancement (PAPE). ${ }^{3}$ The inclusion of sequenced exercises that elicit acute PAPE effects into long-term training programs is also known as complex training (CT). In other terms, CT consists of alternating maximal or near-maximal strengthening exercises with plyometric exercises. ${ }^{4}$ A systematic review with meta-analysis including 33 studies revealed that CT is more effective in improving measures of physical fitness (e.g., muscle strength and linear sprint speed) compared with single-mode plyometric training in individuals aged 14 to 50 years with different physical activity levels. ${ }^{4}$

Further, balance training (BT) can transfer to selected components of physical fitness such as muscle strength and power. ${ }^{5-7}$ For instance, Granacher et al. ${ }^{7}$ investigated the effects of 4 weeks of BT vs. control on body sway, leg-extensor strength, and jump-height in males and females aged 19 years. They reported that BT generated significant improvements in balance, jump-height, and rate of force development of leg extensors. Additionally, Kean et al. ${ }^{8}$ demonstrated significant vertical jump-height improvements following 6 weeks of BT in recreationally active females aged 25 years. Gebel et al. ${ }^{5}$ recently synthesized the literature on the effects of balance training on selected measures of physical fitness in the general youth population and young athletes. In accordance with the principle of training specificity ${ }^{9}$, it was concluded that BT improves static and dynamic balance. In addition, BT-related transfer effects were noted for measures of muscle strength and power. ${ }^{5}$ Taken together, single-mode BT primarily improves balance with beneficial transfer effects on muscle strength, particularly rate of force development of the leg extensors or plantar flexors. ${ }^{5,7}$

A previous longitudinal study showed that combined balance and plyometric exercises have been shown to induce larger improvements in sprint speed and change-of-direction speed performances compared with plyometric training only in male youth aged 12-15 years. ${ }^{10}$ Interestingly, a recent cross-sectional study examined the acute effects of combined balance and strength exercises vs. strength exercises only on subsequent twitch contractile properties, maximum voluntary contraction of the plantar flexors, and jump performance in young female soccer players. ${ }^{11}$ It was found that combined balance and strength exercises but not singlemode strength exercises resulted in acute jump performance improvements. ${ }^{11}$ Based on these findings, sequenced balance and strength exercises may represent a promising means to induce long-term improvements in jump performance if included in a training program such as CT. ${ }^{11}$ To the authors' knowledge, there are no studies available that examined the chronic effects of combining balance exercises with CT compared with CT only on measures of physical fitness (e.g., jump performance) in young athletes. In particular, there is a paucity of research in the CT literature in female participants. ${ }^{4,12}$ Therefore, we aimed to compare the effects of 
8 weeks of balance exercises executed within a CT session and prior to CT exercises (bCT) versus $\mathrm{CT}$ only on measures of physical fitness in young elite female handball players. Our working hypothesis was that performing bCT results in larger measures of physical fitness (e.g., muscle power) adaptations than CT only in young female athletes. ${ }^{6,7,11}$

\section{METHODS}

\section{Experimental design}

A randomized two-groups repeated measures design was used to examine the effects of CT vs. bCT on proxies of muscle power (countermovement jump [CMJ], standing-long-jump [SLJ], reactive-strength index $[R S I]$ ), muscle strength (back half-squat one-maximum repetition [1RM]), dynamic balance (Y-balance test [YBT]), linear sprint speed ( $5 \mathrm{~m}, 10 \mathrm{~m}$, and $20 \mathrm{~m}$ ), and CoD speed (T-test) in young female handball players. The same investigator, who was blinded to group allocation, conducted all measurements. The two intervention arms were realized during the in-season period of the year 2019 (February-March). All tests were scheduled at least 48 hours after the last training session or competitive event. Before testing, a general followed by a specific warm-up routine was performed including 5 min of submaximal running with CoD, 10 min of plyometrics (two submaximal jump exercises of 20 vertical and ten horizontal jumps), dynamic stretching exercises, and five minutes of a sprint-specific warmup. Before and after the training interventions, tests were performed in the following standardized order over four days: anthropometric measurements, balance, and linear sprint tests (day 1), jumping and CoD tests (day 2), and half-squat 1RM test (day 3 ). Two weeks before the start of the study, all athletes participated in two familiarization sessions to become accustomed to the procedure. Participants were used to the applied exercise drills and had achieved good technical competency, through prior training activities.

\section{Participants}

With reference to an intervention study on the effects of CT on jump performance ${ }^{13}$, an a priori power analysis with a type I error rate of 0.05 and $80 \%$ statistical power was computed. The analysis indicated that overall, 20 participants are sufficient to observe significant, medium effects of time (Cohen's $d=0.70$ ) for countermovement jump height. Considering a potential drop out of $10 \%, 24$ healthy young female athletes from the same regional handball club were recruited and randomly allocated either to a CT group $(n=12$, age $=16.9 \pm 0.2$ years; body-mass $=63.7 \pm 5 \mathrm{~kg}$, height $=164.8 \pm 6.3 \mathrm{~cm}$ ) or a bCT group $(\mathrm{n}=12$, age $=16.8 \pm 0.3$ years, body-mass $=63.3 \pm 5.1 \mathrm{~kg}$, and height $=164.0 \pm 6.9 \mathrm{~cm}$ ). Participants' assignment was blinded for participants at pre-test. All participants regularly competed at the national level. They were classified as experienced players with $8.0 \pm 1.2$ years of regular handball training background comprising three to five training sessions per week. The duration of a single training session lasted between 70 and 90 minutes. Players were instructed to miss no more than $10 \%$ of the total number of training and intervention sessions and/or two consecutive sessions. All procedures were approved by the local Institutional Review Committee of the Higher Institute 
of Sport and Physical Education, Ksar Saïd, Tunisia. The study was conducted according to the latest version of the Declaration of Helsinki. Written informed parental consent and participants' assent were obtained before the start of the study. Likewise, all participants and their parents/legal representatives received information on the experimental protocol as well as its potential risks and benefits before its commencement.

\section{Proxies of muscle power}

The CMJ was executed as previously described. ${ }^{14}$ Jump height was recorded using an optoelectric system (Optojump, Microgate, SRL, Bolzano, Italy). A rest period of 1-min was allowed between trials. The best out of three trials was retained for further analysis.

For the SL test, the protocol of Negra et al. ${ }^{15}$ was followed. The horizontal distance between the starting line and the heel of the rear foot was recorded using a tape measure to the nearest $1 \mathrm{~cm}$. A rest period of 1-min between trials was allowed. The best out of three trials was recorded for further analysis.

For the assessment of reactive strength, participants executed five repeated bilateral maximal vertical hops using an Optojump photoelectric system (Microgate, SRL, Bolzano, Italy) for performance assessment. Before testing, youth athletes were instructed to maximize jump height and to shorten ground contact time. The first jump was not counted and the four remaining jumps were averaged for the calculation of RSI using the following formula: $R S I=$ jump height $(\mathrm{mm})$ / ground contact time (ms). A rest period of 5-min between trials was allowed. The best out of two trials was recorded for further analysis.

\section{Maximal strength}

The back half-squat exercise was used to determine each individual's maximal leg extensor strength according to the protocol proposed by Faigenbaum et al. ${ }^{16}$ The 1RM represents the maximum weight that can be lifted by a participant throughout the full range of motion $\left(90^{\circ}\right.$ knee flexion). Before attempting a 1RM trial, participants performed five to six repetitions at a relatively light load ( $\sim 40 \%$ of their last $1 \mathrm{RM}$ test). Thereafter, three to four repetitions were performed at a heavier load ( $70 \%$ of their estimated 1RM). Finally, a single repetition was conducted with a load corresponding to $95 \%$ of the estimated 1RM. Afterward, participants attempted a single repetition with the perceived 1RM load. If this load was lifted with proper technique, the load was increased by another 1.0 to $2.5 \mathrm{~kg}$, and the participant attempted another repetition. Failure was defined as a lift falling short of the full range of motion on at least two trials with a 2 min rest between trials. The 1RM was typically determined within four to five trials.

\section{Dynamic balance}

Dynamic balance was measured using the Y-balance test using tape measures on the floor as previously outlined ${ }^{15}$. Before testing, participants' left and right leg length were assessed in supine lying position by measuring the distance from the anterior superior iliac spine to the most distal aspect of the medial malleolus. The best score of three successful attempts 
expressed as the maximal reach distance in centimeters for each direction was retained for further analysis with no more than six attempts. The composite score (CS) was calculated as follows: $\mathrm{CS}=[$ (maximum anterior reach distance + maximum posteromedial reach distance + maximum posterolateral reach distance)/(leg-length $\times 3)] \times 100$.

\section{Linear sprint speed}

The performance of a $20 \mathrm{~m}$ linear sprint with split sprint times of $5 \mathrm{~m}$ and $10 \mathrm{~m}$ were recorded using an infrared photocell system (Microgate, SRL, Bolzano, Italy). The between-trial recovery time was 3-min. The best performance out of two trials was used for further analysis.

\section{Change-of-direction speed}

The T-test was conducted as described previously. ${ }^{14}$ The final performance outcome is expressed as the time needed to complete the test. This was assessed using a single beam infrared photocell device (Microgate SRL, Bolzano, Italy). Each participant performed two trials with a 3-min rest between each. The fastest recorded time was used for further analysis.

\section{Characteristics of the training programs}

During the study, all participants were not engaged in any kind of activities other than their regular handball training routine. Details of both $\mathrm{CT}$ and $\mathrm{BCT}$ programs are displayed in Table 1. Both training interventions were conducted each Tuesday and Thursday for 8 weeks. A standardized 8-to-15-min warm-up was completed before every training session for the two experimental groups. The warm-up included low intensity running, coordination exercises, dynamic movements (lunges and skips), sprints, and dynamic stretching of the lower-limb muscles. The training session lasted on average 45-min. The number of repetitions, sets, and the complexity of the exercises were progressively increased over the training period. Specifically, the CT group performed 3 sets of back half squat, 8 repetitions per set at $80 \%$ $1 \mathrm{RM}$, and a 2-min of rest in-between-sets followed by 3-min recovery. Thereafter, athletes performed 3-to-4 sets with 6-to-10 repetitions per set of CMJs with a $90 \mathrm{~s}$ rest in-between sets. ${ }^{17}$ The bCT group performed the same sequence of strength and plyometric exercises preceded by 3 sets, $40 \mathrm{~s}$ each, and $20 \mathrm{~s}$ of rest in-between sets of balance exercises. ${ }^{11}$ There was no rest between balance and strength exercises. ${ }^{11}$ Training time for balance amounted to approximately $2.6 \mathrm{~min}$ per session which equalled $41.6 \mathrm{~min}$ of balance training in bCT over the entire intervention period. However, given that the interventions were conducted in replacement of some technical/tactical handball exercises, total training volume was similar for both groups. Specifically, the balance training consisted of a double-leg stance on a balance board under two conditions; eyes opened (the first 4 weeks) and closed (the second four weeks). During the performance of CMJs, participants were instructed to jump as high as possible in a non-continuous manner (3-to-5 s in-between reps). The back half-squat 1RM was reassessed after four weeks of training and the 1RM load was adjusted accordingly for the rest of the training intervention. 


\section{Statistical Analyses}

Data were tested and confirmed for normal distribution using the Shapiro-Wilks test. Data were presented as group mean values with standard deviations. Baseline between-group differences were computed using independent t-tests. To establish the effect of the training interventions on the dependent variables, a 2 (group: bCT and CT) $\times 2$ (time: pre, post) repeated measures ANOVA was computed. In the case of significant group $\times$ time interactions, group-specific repeated measure ANOVAs (time: pre, post) were used to determine withingroup changes. Additionally, effect sizes (ES) were determined by converting partial etasquared to Cohen's $d$. Effect sizes were classified as small $(d<0.50)$, medium $(0.50 \leq d<0.80)$, and large $(d \geq 0.80) .{ }^{18}$ Within-session test-retest reliability was assessed during the pre-test using the intraclass correlation coefficients (ICCS) and the standard error of measurement (SEM) expressed as coefficient of variation. ${ }^{19}$ The level of statistical significance was established as $p \leq 0.05$. The SPSS 26.0 (SPSS Inc., Chicago, IL, USA) was used for statistical analyses.

\section{RESULTS}

All participants received treatments as allocated. One participant in the bCT group dropped out because she left the handball training center for personal reasons (Figure 1). Thus, 23 athletes completed the training program. The adherence rate to training was $97 \%$ for both groups. Participants reported no training- or test-related injuries during the study. Table 2 depicts the between-trials reliability of the different measures of physical fitness. All ICC values were $\geq 0.80$ (from 0.80 to 0.97 ) and SEM values $<5 \%$ (from 0.78 to $4.38 \%$ ) indicating good reliability for all physical fitness parameters (Table 2). Table 3 displays test data for all measures of physical fitness at pre- and post-intervention. There were no significant betweengroup differences at baseline (Table 3 ).

Figure 1 near here

Table 2 near here

Table 3 near here

\section{Proxies of muscle power}

A significant group $\times$ time interaction effect was observed for RSI $(d=1.0, p<0.05)$. The posthoc analysis showed a significant pre-to-post-test performance improvement for the CT group only ( $p<0.05, d=0.7$; Figure 2 ). Further, a significant main effect of time was observed for the CMJ $(d=1.5, p<0.01)$ and the SLJ $(d=1.7, p<0.01)$ tests with no significant group $\times$ time interactions ( $d=0.6$ and 0.4 for CMJ and $S \sqcup$, respectively, $p>0.05$ ).

Figure 2 near here

Muscle strength 
A significant main effect of time was noted for the 1 RM back half-squat test $(d=3.1, p<0.001)$, whereas no significant group $\times$ time interaction, was observed $(d=0.4, p>0.05)$.

\section{Dynamic balance}

A significant group $\times$ time interaction was observed $(d=1.3, p<0.01)$. The post-hoc analysis showed a significant increase in Y-balance test score from pre- to post-test for the bCT group only $(d=0.7, p<0.05$; Figure 3$)$.

\section{Speed}

Linear sprint speed

There was no significant group $\times$ time interaction or main effect of time for any of the sprint intervals $(0-5 \mathrm{~m}, 0-10 \mathrm{~m}$, and $0-20 \mathrm{~m})(d=0.1-0.8, p>0.05)$.

\section{Change-of-direction speed}

For the T-test, a significant main effect of time was observed $(d=3.1, p<0.001)$. However, group $\times$ time interaction failed to reach the significance level $(d=0.4, p>0.05)$.

Figure 3 near here

\section{Discussion}

The main outcomes of our study indicated that both training types were effective in improving measures of physical fitness in young elite female handball players when combined with regular handball training. However, if the goal is to improve dynamic balance, bCT seems to be more effective than CT only.

The purpose of CT programs is to translate acute PAPE to more efficient and chronic training gains. 3,4 Various physiological effects, e.g., warm-up related changes in muscle temperature, metabolism, baseline oxygen consumption, muscle activation, motor learning, and even subjects' psychological state have been reported to induce acute and transient PAPE. ${ }^{3}$ The findings of the present study indicate that CT as well as BCT induced significant gains in measures of maximal strength (i.e., back half-squat 1RM), muscle power (i.e., CMJ, SLJ), and CoD speed (i.e., T-test). Recently, Hammami et al. ${ }^{20}$ studied the effects of 10 weeks of CT on measures of physical fitness in a similar cohort of young female handball players aged 16 years. They reported significant improvements in maximal strength (i.e., half-squats 1RM), jump performance (i.e., squat jump, CMJ, CMJ aided-arm, and five-jump test), sprint speed (i.e., 10-m, 20-m, 30-m) and CoD speed (i.e., T-half test, modified Illinois test) following CT ( $\Delta 4$ $19 \%)$ compared with a control group. Furthermore, Bauer et al. ${ }^{4}$ systematically analyzed the literature for chronic effects of CT studies and demonstrated that CT is effective in improving measures of physical fitness such as maximal strength, jump performance, and linear sprint speed in males and females aged 14 to 50 years. Interestingly, improvements in maximal 
strength and linear sprint speed following CT appear to be even superior when compared to gains following traditional training programs (e.g., single-mode strength or plyometric training). ${ }^{4}$ Considering that greater maximal strength is associated with higher sport-specific performances, the authors highlighted the importance of CT programs as an effective training strategy. ${ }^{4}$ In this regard, it can be speculated that strength gains following CT and bCT may translate to handball performance in young female handball players. ${ }^{21}$ In contrast to the findings of Hammami et al. ${ }^{20}$ and Bauer et al. ${ }^{4}$, our findings revealed no significant performance changes in any of the linear sprint speed intervals (i.e., 0-5 m, 0-10 m, and 0-20 $\mathrm{m}$ ) following both training interventions. The reason for the absence of beneficial effects on linear sprint speed following BCT and CT programs is not clear. Of note, the baseline sprint performance measures of our participants over the $20 \mathrm{~m}$ distance were higher in the $\mathrm{bCT}$ and CT group (9\%) compared with similar participants in the study of Hammami et al. ${ }^{20}$. In accordance with the law of diminishing returns, ${ }^{22}$ our participants could have been less likely to achieve significant improvements in sprint performance after training due to the higher fitness level. Additionally, it appears that the difference in the applied methodology between our study and the study of Hammami et al. ${ }^{20}$ (e.g., 8 weeks vs. 10 weeks of training, absence vs. presence of sprint tasks; presence vs. absence of horizontal jumping tasks, and only back half-squat vs. back half-squat, calf raise, and thigh press, respectively) has led to different outcomes. Given that studies addressing the effects of CT programs on sprint performance in females are scarce, ${ }^{4}$ future research is warranted.

Interestingly our findings indicate specific adaptations after eight weeks of CT versus BCT in young female handball players. More precisely, we found significant pre- to post-test increases in the Y-balance test score for the bCT group only. Hammami et al. ${ }^{20}$ did not report any significant improvements in static (stork-balance-test) and dynamic (Y-balance test) balance following 8 weeks of CT in young female handball players, which is in agreement with the current outcomes. Yet, with the principle of training specificity in mind, ${ }^{23}$ it appears that performing a block of balance exercises before CT has contributed to the improvement of dynamic balance performance in young female handball players in our study. Further, we found a significant medium-sized pre-to-post improvement in RSI for the CT group only. This finding is partly in line with the study of Faude et al. ${ }^{24}$, who reported significant increments in $\mathrm{RSI}$, following $\mathrm{CT}$, compared with a control condition in male, high-level amateur soccer players. Additionally, Makhlouf et al. ${ }^{25}$ showed that adding balance exercises before plyometric training programs for eight weeks does not necessarily increase RSI values in young male soccer players. Thus, it seems that performing balance exercises before CT hampers reactive strength gains in our study. From a physiological perspective, balance exercises reduce spinal reflex activity in the short- and long-term. ${ }^{26}$ However, spinal reflexes contribute to stretch-shortening cycle exercises such as drop jumps. ${ }^{27,28}$ It can be speculated that compromised reactive strength gains following bCT compared with CT were attributed to the reduced spinal reflex activities during plyometric exercises. Nevertheless, future studies with mechanistic approaches are needed to confirm this hypothesis. 
This study has some limitations that have to be acknowledged. First, we could not include an active control group owing to the limited number of participants available. However, the main purpose of this study was not to explore the general effectiveness of CT on measures of physical fitness. This has already been addressed in previous studies. ${ }^{4,12,29}$ The goal was rather to examine the specific effects of adding balance exercises prior to CT within a training session vs. CT only. Therefore, to answer such a research question, a control group is not a decisive element. Second, the limited number of bCT induced changes beyond those of CT (only for dynamic balance) could be due to the overall low dosage of balance exercises. Future studies should consider a higher dosage of balance exercises prior to CT. Third, it would have been interesting to contrast training-related adaptations between males and females to improve results' generalizability. This should constitute the purpose of future research. Finally, all analyzed fitness measures were performance-related. We acknowledge that this study does not reveal any information on the underlying physiological mechanisms. Future studies should, therefore, assess physiological parameters for instance electromyographic data to obtain insight into the underlying mechanisms.

\section{Practical applications}

Both bCT and CT interventions induced similar effects on components of physical fitness in young female handball players. However, if the goal is to additionally improve balance, coaches and strength and conditioning professionals should add a block of balance exercises prior to CT programs.

\section{Conclusions}

The main findings of this study showed that $\mathrm{BCT}$ and CT were effective in improving measures of physical fitness in young elite female handball players. More specifically, to improve dynamic balance, bCT seems to be more effective than CT. Future studies may apply different dosages of balance exercises within bCT to find out whether the observed effects are dependent on the dose of balance exercise.

\section{References}

1. Saez-Saez de Villarreal E, Requena B, Newton RU. Does plyometric training improve strength performance? A meta-analysis. Journal of science and medicine in sport. 2010;13(5):513-522.

2. Lesinski M, Prieske O, Granacher U. Effects and dose-response relationships of resistance training on physical performance in youth athletes: a systematic review and meta-analysis. British journal of sports medicine. 2016;50(13):781-795.

3. Prieske O, Behrens M, Chaabene H, Granacher U, Maffiuletti NA. Time to Differentiate Postactivation "Potentiation" from "Performance Enhancement" in the Strength and Conditioning Community. Sports Medicine. 2020. 
4. Bauer P, Uebellacker F, Mitter B, et al. Combining higher-load and lower-load resistance training exercises: A systematic review and meta-analysis of findings from complex training studies. Journal of science and medicine in sport. 2019;22(7):838-851.

5. Gebel A, Prieske O, Behm DG, Granacher U. Effects of Balance Training on Physical Fitness in Youth and Young Athletes: A Narrative Review. Strength \& Conditioning Journal. 2020;Publish Ahead of Print.

6. Behm D, Colado JC. The effectiveness of resistance training using unstable surfaces and devices for rehabilitation. Int J Sports Phys Ther. 2012;7(2):226-241.

7. Granacher U, Gollhofer A, Kriemler S. Effects of balance training on postural sway, leg extensor strength, and jumping height in adolescents. Res Q Exerc Sport. 2010;81(3):245-251.

8. Kean CO, Behm DG, Young WB. Fixed foot balance training increases rectus femoris activation during landing and jump height in recreationally active women. Journal of sports science \& medicine. 2006;5(1):138.

9. Morrissey MC, Harman EA, Johnson MJ. Resistance training modes: specificity and effectiveness. Medicine and science in sports and exercise. 1995;27(5):648-660.

10. Chaouachi A, Othman AB, Hammami R, Drinkwater EJ, Behm DG. The combination of plyometric and balance training improves sprint and shuttle run performances more often than plyometric-only training with children. Journal of strength and conditioning research. 2014;28(2):401-412.

11. Prieske O, Maffiuletti NA, Granacher U. Postactivation Potentiation of the Plantar Flexors Does Not Directly Translate to Jump Performance in Female Elite Young Soccer Players. Frontiers in physiology. 2018;9(276).

12. Cormier P, Freitas TT, Rubio-Arias JÁ, Alcaraz PE. Complex and Contrast Training: Does Strength and Power Training Sequence Affect Performance-Based Adaptations in Team Sports? A Systematic Review and Meta-analysis. The Journal of Strength \& Conditioning Research. 2020.

13. Veliz RR, Suarez-Arrones L, Requena B, Haff GG, Feito J, Sáez de Villarreal E. Effects of incompetitive season power-oriented and heavy resistance lower-body training on performance of elite female water polo players. Journal of strength and conditioning research. 2015;29(2):458-465.

14. Negra Y, Chaabene H, Hammami M, et al. Agility in Young Athletes: Is It a Different Ability From Speed and Power? Journal of strength and conditioning research. 2017;31(3):727-735.

15. Negra Y, Chaabene H, Sammoud S, et al. Effects of Plyometric Training on Physical Fitness in Prepuberal Soccer Athletes. International journal of sports medicine. 2017;38(5):370-377.

16. Faigenbaum AD, Westcott WL, Loud RL, Long $C$. The effects of different resistance training protocols on muscular strength and endurance development in children. Pediatrics. 1999;104(1):e5-e5.

17. Dobbs WC, Tolusso DV, Fedewa MV, Esco MR. Effect of Postactivation Potentiation on Explosive Vertical Jump: A Systematic Review and Meta-Analysis. Journal of strength and conditioning research. 2019;33(7):2009-2018.

18. Cohen J. Statistical power analysis for the behaviors science.(2nd). New Jersey: Laurence Erlbaum Associates, Publishers, Hillsdale. 1988.

19. Weir JP. Quantifying test-retest reliability using the intraclass correlation coefficient and the SEM. The Journal of Strength \& Conditioning Research. 2005;19(1):231-240.

20. Hammami M, Gaamouri N, Aloui G, Shephard RJ, Chelly MS. Effects of a Complex StrengthTraining Program on Athletic Performance of Junior Female Handball Players. International journal of sports physiology and performance. 2019;14(2):163-169.

21. Granados $C$, Izquierdo M, Ibáñez J, Ruesta M, Gorostiaga EM. Are there any differences in physical fitness and throwing velocity between national and international elite female handball players? Journal of strength and conditioning research. 2013;27(3):723-732.

22. Hackfort D, Schinke RJ, Strauss B. Dictionary of sport psychology: sport, exercise, and performing arts. Academic Press; 2019. 
23. Behm DG. Neuromuscular implications and applications of resistance training. Journal of

24. Faude O, Roth R, Di Giovine D, Zahner L, Donath L. Combined strength and power training in high-level amateur football during the competitive season: a randomised-controlled trial. Journal of sports sciences. 2013;31(13):1460-1467.

25. Makhlouf I, Chaouachi A, Chaouachi M, Ben Othman A, Granacher U, Behm DG. Combination of agility and plyometric training provides similar training benefits as combined balance and plyometric training in young soccer players. Frontiers in physiology. 2018;9:1611.

26. Taube W, Gruber M, Gollhofer A. Spinal and supraspinal adaptations associated with balance training and their functional relevance. Acta physiologica (Oxford, England). 2008;193(2):101116.

27. Komi PV, Gollhofer A. Stretch Reflexes Can Have an Important Role in Force Enhancement during SSC Exercise. 1997;13(4):451.

28. Taube W, Leukel C, Gollhofer A. How neurons make us jump: the neural control of stretchshortening cycle movements. Exercise and sport sciences reviews. 2012;40(2):106-115.

29. Freitas TT, Martinez-Rodriguez A, Calleja-González J, Alcaraz PE. Short-term adaptations following Complex Training in team-sports: A meta-analysis. PloS one. 2017;12(6):e0180223.

30. Seo DI, Kim E, Fahs CA, et al. Reliability of the one-repetition maximum test based on muscle group and gender. Journal of sports science \& medicine. 2012;11(2):221-225. 
Table 1: Characteristics of the two training interventions

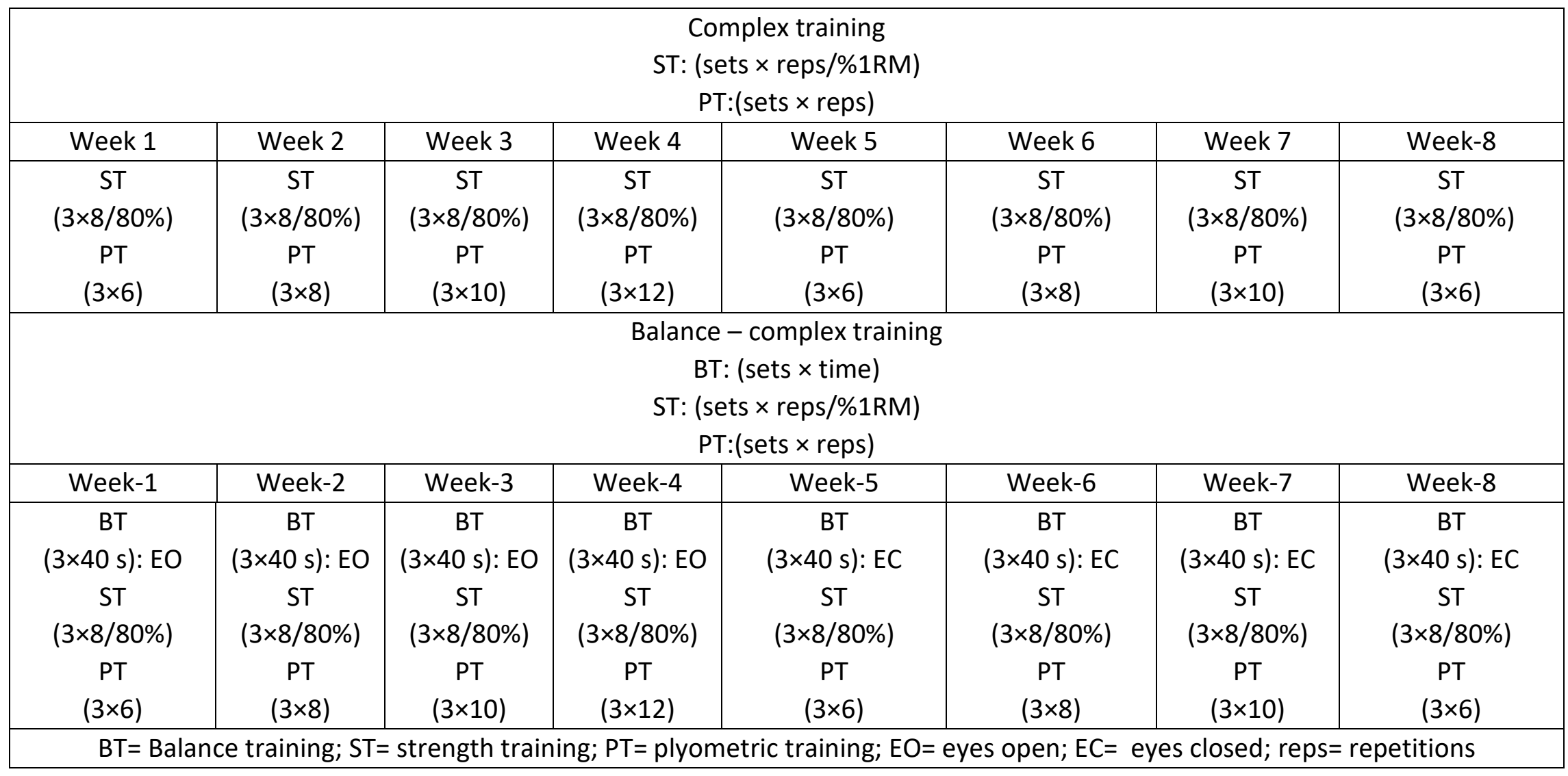




\begin{tabular}{|c|c|c|c|c|}
\hline Physical fitness measure & $\begin{array}{l}\text { Intraclass correlation coefficient } \\
\text { (ICC) }\end{array}$ & $\begin{array}{l}\text { Standard error of } \\
\text { measurement (SEM) }\end{array}$ & SEM (\%) & $\begin{array}{l}469 \\
470\end{array}$ \\
\hline Muscle power & & & & 471 \\
\hline CMJ & 0.96 & 0.46 & 2.09 & 472 \\
\hline SLJ & 0.97 & 0.03 & 2.13 & 473 \\
\hline RSI & 0.88 & 0.02 & 4.38 & 474 \\
\hline Muscle strength & & & & 475 \\
\hline Back half squat $1 \mathrm{RM}$ & $0.97^{*}$ & & - & 476 \\
\hline Dynamic balance & & & & 410 \\
\hline Y-balance test & 0.80 to 0.919 & 2.61 to 3.029 & 3.16 to 3.609 & 477 \\
\hline Speed & & & & 478 \\
\hline $5 \mathrm{~m}$ & 0.92 & 0.04 & 2.8 & 479 \\
\hline $10 \mathrm{~m}$ & 0.94 & 0.02 & 0.78 & 480 \\
\hline $20 \mathrm{~m}$ & 0.97 & 0.10 & 2.85 & 481 \\
\hline Change of direction speed & & & & 482 \\
\hline T-test & 0.91 & 0.38 & 3.22 & 483 \\
\hline
\end{tabular}

CMJ: countermovement jump; SL: standing long jump; RSI: reactive strength index; 1RM: one-repetition maximum; *from the study of Seo et al. ${ }^{30}$; 9 range 
Table 3: Group-specific changes in measures of physical fitness from pre-to-post.

\begin{tabular}{|c|c|c|c|c|c|c|c|c|c|c|c|}
\hline & \multicolumn{4}{|c|}{$C T(n=12)$} & \multicolumn{4}{|c|}{$\mathrm{bCT}(\mathrm{n}=11)$} & \multirow{2}{*}{\multicolumn{3}{|c|}{$\frac{\text { ANOVA }}{p \text {-value (ES) }}$}} \\
\hline & \multicolumn{2}{|c|}{ Pretest } & \multicolumn{2}{|c|}{ Posttest } & \multicolumn{2}{|c|}{ Pretest } & \multicolumn{2}{|c|}{ Posttest } & & & \\
\hline & M & SD & M & SD & $M$ & SD & $M$ & SD & Time & Group & Group $\times$ Time \\
\hline \multicolumn{12}{|c|}{ Muscle power } \\
\hline $\mathrm{RSI}(\mathrm{mm} / \mathrm{ms})$ & 0.7 & 0.4 & 0.8 & 0.4 & 0.8 & 0.4 & 0.8 & 0.4 & $0.305(0.5)$ & $0.909(0.1)$ & $0.033(1.0)$ \\
\hline $\mathrm{SLJ}(\mathrm{m})$ & 1.6 & 0.2 & 1.7 & 0.2 & 1.6 & 0.2 & 1.7 & 0.2 & $0.001(1.7)$ & $0.840(0.1)$ & $0.435(0.4)$ \\
\hline $\mathrm{CMJ}(\mathrm{cm})$ & 22.5 & 5.1 & 24.1 & 5.2 & 21.8 & 5.7 & 22.5 & 5.6 & $0.002(1.5)$ & $0.607(0.2)$ & $0.169(0.6)$ \\
\hline \multicolumn{12}{|c|}{ Muscle strength } \\
\hline 1 RM back half-squat (kg) & 96.9 & 15.4 & 104.0 & 13.5 & 79.5 & 15.1 & 88.7 & 11.5 & $0.001(3.1)$ & $0.011(1.2)$ & $0.358(0.41)$ \\
\hline \multicolumn{12}{|c|}{ Dynamic balance } \\
\hline Y-balance test & 93.7 & 8.0 & 93.2 & 8.2 & 90.20 & 90.2 & 91.1 & 9.7 & $0.345(0.4)$ & $0.445(0.3)$ & $0.007(1.3)$ \\
\hline \multicolumn{12}{|c|}{ Linear sprint speed } \\
\hline 5-m sprint (s) & 1.3 & 0.1 & 1.3 & 0.1 & 1.3 & 0.1 & 1.3 & 0.1 & $0.643(0.2)$ & $0.687(0.2)$ & $0.749(0.1)$ \\
\hline 10-m sprint (s) & 2.2 & 0.2 & 2.1 & 0.1 & 2.1 & 0.1 & 2.1 & 0.1 & $0.094(0.8)$ & $0.929(0.0)$ & $0.121(0.7)$ \\
\hline 20-m sprint (s) & 3.5 & 0.2 & 3.5 & 0.2 & 3.5 & 0.2 & 3.5 & 0.2 & $0.711(0.2)$ & $0.891(0.1)$ & $0.657(0.2)$ \\
\hline \multicolumn{12}{|c|}{ Change of direction speed } \\
\hline T-test (s) & 11.6 & 0.6 & 11.1 & 0.1 & 11.6 & 0.9 & 11.2 & 0.9 & $0.001(3.1)$ & $0.888(0.1)$ & $0.352(0.4)$ \\
\hline
\end{tabular}

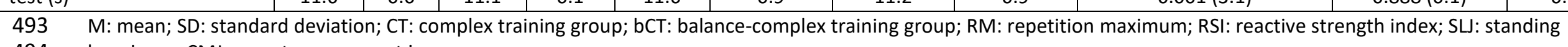

494 long jump; CMJ: countermovement jump;

495

496

497

498

499

500 
Figure 1: Flow chart of the progress through the phases of the study according to the CONSORT statements.

\section{Enrollment}

\section{CT group}

Allocated to intervention $(n=12)$

- Received allocated intervention $(n=12)$

- Did not receive allocated intervention (give reasons) $(n=0)$
Assessed for eligibility $(n=24)$

Excluded $(\mathrm{n}=0)$

- Not meeting inclusion criteria $(n=0)$

- Declined to participate $(n=0)$

- Other reasons $(n=0)$

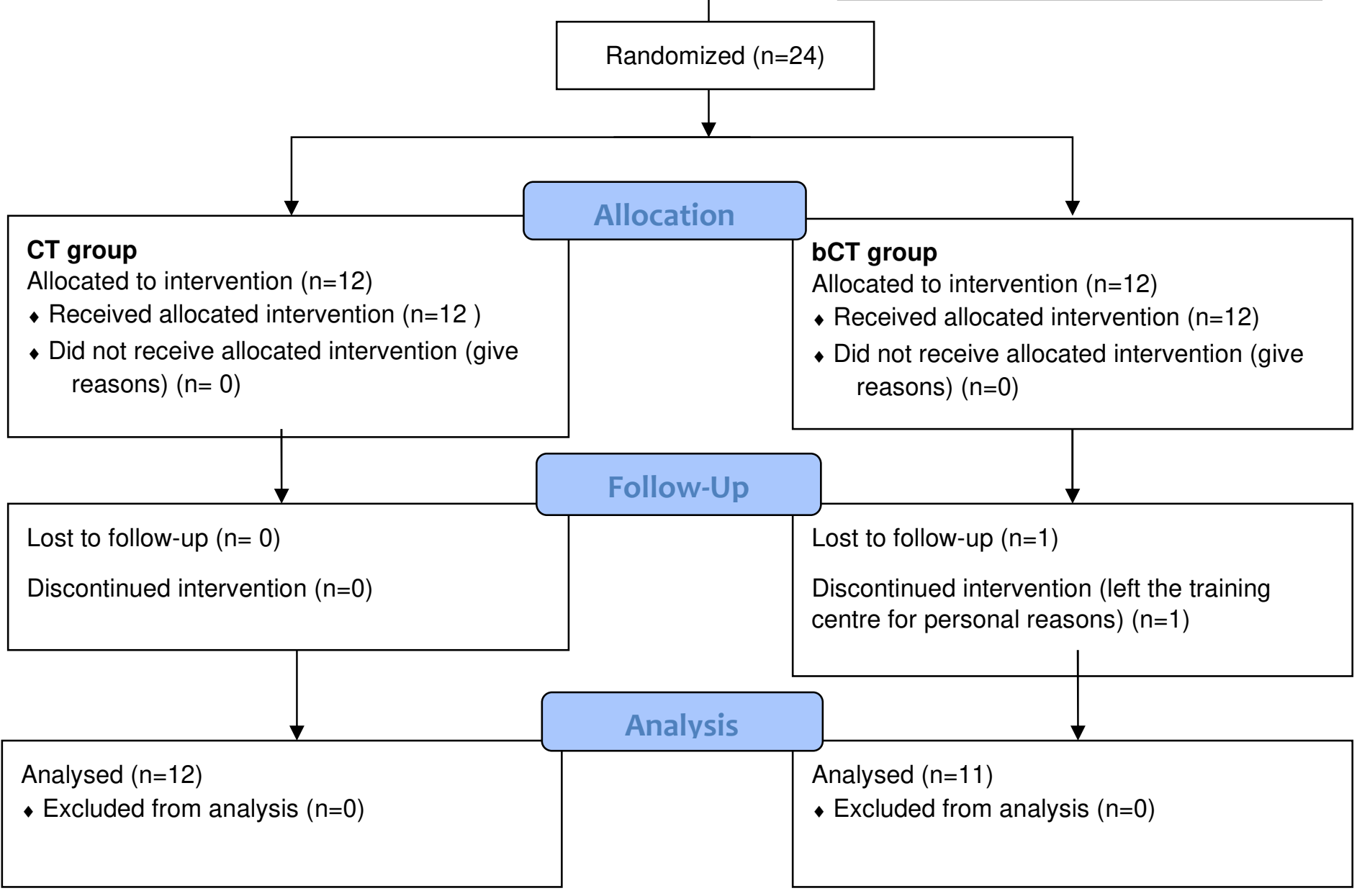




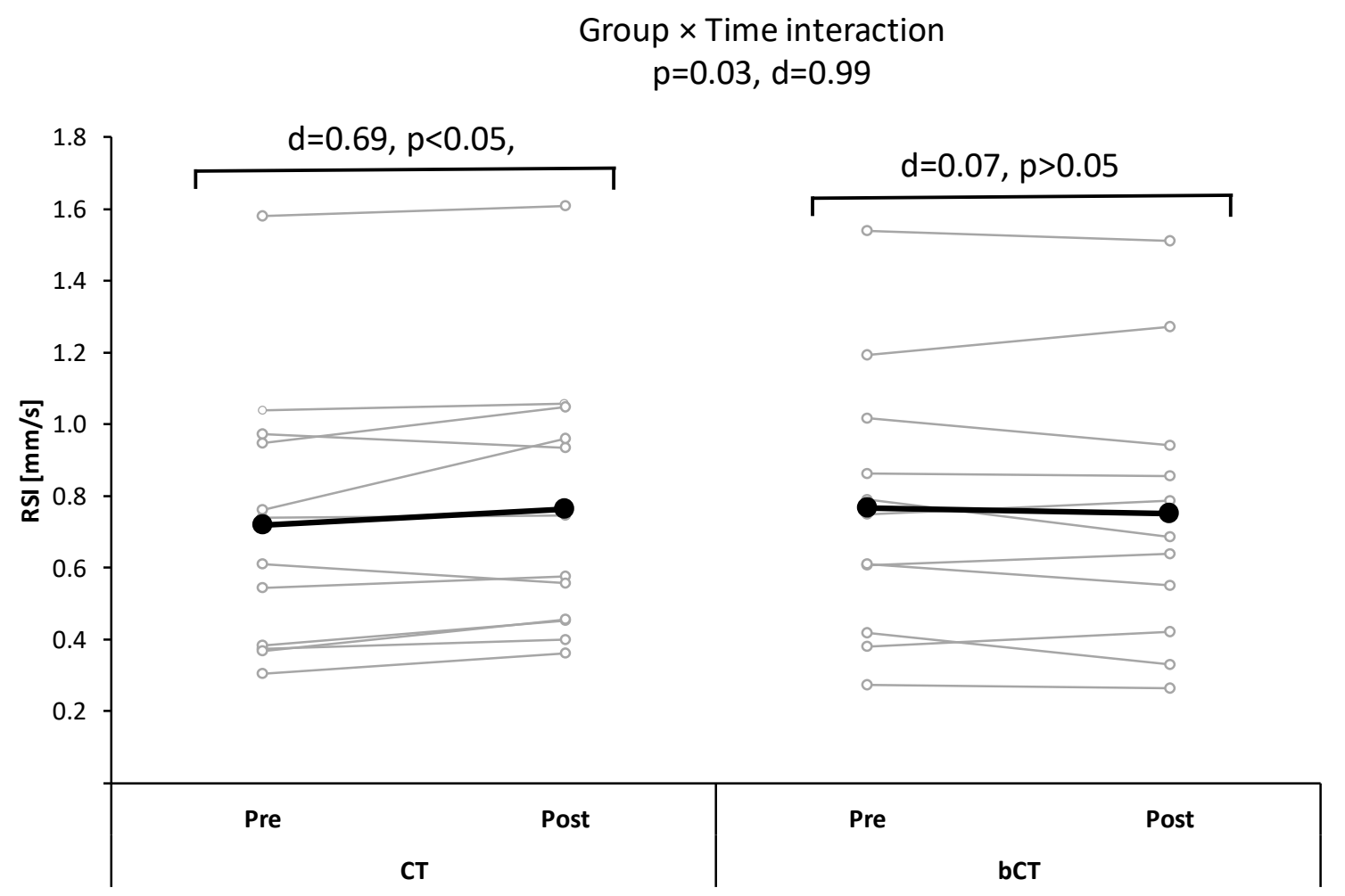

505

506 Figure 2: Changes in reactive strength index (RSI) following 8 weeks of complex training (CT)

507 or combined balance and complex training (bCT) in young female handball players. 


\section{Group $\times$ Time interaction}

$p<0.01, d=1.32$

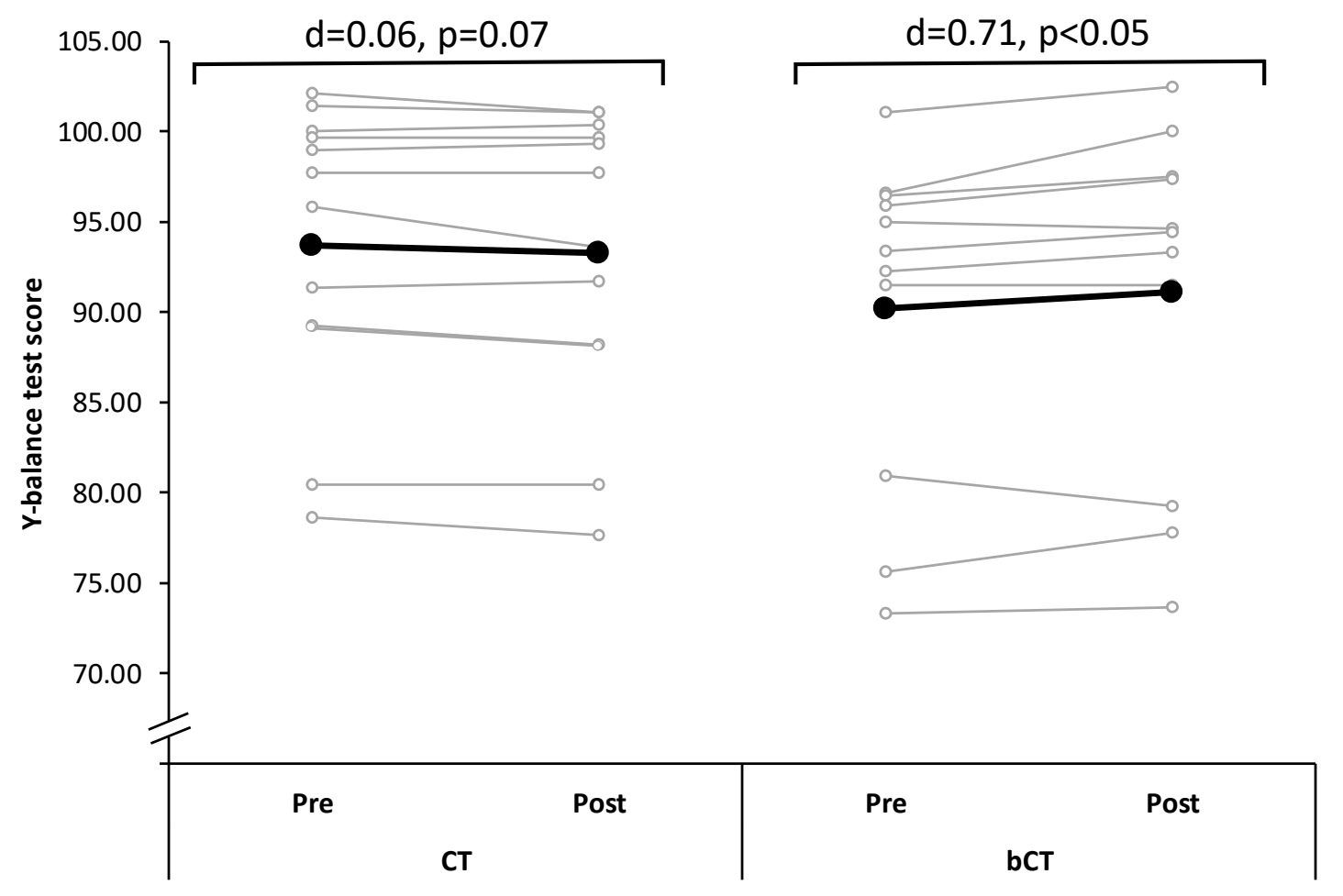

513

514 Figure 3: Changes in Y-balance test score following 8 weeks of complex training (CT) or 515 combined balance and complex training ( $\mathrm{bCT}$ ) in young female handball players.

Grey line: Pre-to-post individual outcomes 